\title{
THE USE OF EXTENDED VALUED NORMAL FORMS IN REMOVING A BOUNDEDNESS CONDITION ON AN OPERATOR INEQUALITY
}

\author{
C. C. BROWN
}

(Communicated by John B. Conway)

\begin{abstract}
The cone of nonnegative extended valued normal forms, defined on the nonnegative bounded symmetric operators in a Hilbert space, is discussed in connection with generalising a known inequality to the unbounded case.

The cone of normal forms seems to offer a possibility for seeing unbounded operators in a more general perspective.
\end{abstract}

1. It was proved in [2] that an inequality which was already known for restricted cases is also valid for general $\sigma$-finite measure spaces. The inequality seems to suggest a probabilistic interpretation of irreversible entropy increase which could be of use in the theory of statistical mechanics. If such an interpretation is justified, then it is natural to look for a corresponding operator inequality, suitable for interpreting entropy increase in quantum statistics. An inequality of G. Lindblad [5], then already in the literature, seems to fulfill the requirements up to a certain point. The present paper is motivated by questions which arise upon comparing the inequality in [2] with that of Lindblad. In order to be more precise, we state the former inequality in a way.most suitable for comparison with Lindblad's, considering the case where only one measure space $(\Omega, \mathfrak{A}, \mu)$ is involved.

In the terminology used in [2], let $\mathscr{L}$ be the convex cone of nonnegative extended real-valued measurable functions on $\Omega, \mathscr{L}_{\mu}$ the convex cone of equivalence classes $\bmod \mu$ of elements of $\mathscr{L}$, and $T^{\prime}$ (continuing to use the notation in [2]) any monotone continuous positive linear map from $\mathscr{L}_{\mu}$ into itself. If

and

$$
\int_{\Omega}\left[T^{\prime} g\right] \mu(d \omega)=\int_{\Omega} g \mu(d \omega) \quad\left(g \in \mathscr{L}_{\mu}\right)
$$

$$
T^{\prime} e=e
$$

where $e \in \mathscr{L}_{\mu}$ with $e(\omega)=1$ for $\mu$-almost all $\omega \in \Omega$, then for any finite-valued convex function $\phi$ defined on $[0, \infty)$ and any $\rho \in \mathscr{L}_{\mu}$ with $\int \rho \mu(d \omega)<\infty$, the finiteness of $\int \phi_{+}(\rho) \mu(d \omega)^{1}$ implies the finiteness of $\int \phi_{+}\left(T^{\prime} \rho\right) \mu(d \omega)$, and

$$
\int_{\Omega} \phi\left(T^{\prime} \rho\right) \mu(d \omega) \leq \int_{\Omega} \phi(\rho) \mu(d \omega)
$$

if the latter integrals exist in the extended sense.

Received by the editors March 9, 1987 and, in revised form, May 18, 1987.

1980 Mathematics Subject Classification (1985 Revision). Primary 15A63, 46L50; Secondary $82 \mathrm{~A} 15$.

Key words and phrases. Normal forms, operators in Hilbert space.

${ }^{1}$ The symbols, $\phi_{+}$and $\phi_{-}$denote the positive and negative parts respectively of the function $\phi$. 
We state Lindblad's theorem in a slightly altered form for comparison, introducing first the necessary Hilbert space terminology. Let $\mathscr{H}$ be a separable Hilbert space, $B$ a bounded selfadjoint operator in $\mathscr{H}$. We define the trace of $B$ only if the positive part, $B_{+}$, or the negative part, $B_{-}$, has a finite trace as defined in the usual sense. We then say, $\operatorname{tr}[B]=\operatorname{tr}\left[B_{+}\right]-\operatorname{tr}\left[B_{-}\right]$. If $K(\mathscr{H})$ is the convex cone of nonnegative bounded selfadjoint operators in $\mathscr{H}$ and $A$ and $B$ elements of $K(\mathscr{H})$, then $A \geq B$ means, as is usual, $A-B \in K(\mathscr{H})$. If $S: K(\mathscr{H}) \rightarrow K(\mathscr{H})$ is a positive linear map, we call $S$ monotone continuous if, for every sequence of elements, $A_{1} \leq A_{2} \leq A_{3} \leq \cdots$, of $K(\mathscr{H})$ with sup $A_{i} \in K(\mathscr{H})$, one has $S\left(\sup A_{i}\right)=\sup S\left(A_{i}\right)$. In the following we continue to use the symbol $T^{\prime}$ instead of $T$ for the transformation.

THEOREM. Let $\phi$ be a bounded convex function defined on the interval, $[0, \infty) \in$ $R^{1}$ and suppose that the positive linear map $T^{\prime}: K(\mathscr{H}) \rightarrow K(\mathscr{H})$ is monotone continuous. If

$$
\operatorname{tr}\left[T^{\prime} B\right]=\operatorname{tr}[B] \quad(B \in K(\mathscr{H}))
$$

and

$$
T^{\prime} I=I
$$

where $I$ is the identity operator, then, for $J \in K(\mathscr{H})$ with $\operatorname{tr}[J]<\infty$, the finiteness of $\operatorname{tr}\left[\phi_{+}(J)\right]$ implies the finiteness of $\operatorname{tr}\left[\phi_{+}\left(T^{\prime} J\right)\right]$. If $\operatorname{tr}\left[\phi\left(T^{\prime} J\right)\right]$ and $\operatorname{tr}[\phi(J)]$ both exist, then

$$
\operatorname{tr}\left[\phi\left(T^{\prime} J\right)\right] \leq \operatorname{tr}[\phi(J)]
$$

If one assumes with Lindblad that $\phi(0)=0$, then the condition $T^{\prime}(I)=I$ can be replaced by $T^{\prime}(I) \leq I$ with practically no alternation of the proof.

Since the real variables inequality in [2] contains no boundedness conditions on the function, $\phi$, the appearance of such conditions in Lindblad's inequality seems to suggest the possibility of finding a generalisation of it. Attempts to generalise in a trivial way seem to lead to the necessity of defining $T^{\prime} \phi(J)$ for unbounded $\phi$, which is even problematical if $\phi$ is nonnegative. One possibility for overcoming this difficulty is to find a cone that is bigger than $K(\mathscr{H})$ in which there is more freedom for defining what is necessary. The appropriate cone seems to be that of extended valued normal forms on $K(\mathscr{H})$.

We define a normal form, $\nu: K(\mathscr{H}) \rightarrow[0, \infty]$, in a way similar to that found in [3] for the finite-valued case, by demanding positive linearity and monotone continuity, i.e. $\nu$ is a normal form if

(1) $\nu(\alpha A+\beta B)=\alpha \nu(A)+\beta \nu(B)$ for all $A$ and $B$, elements of $K(\mathscr{H})$, and all $\alpha$ and $\beta$, elements of $[0, \infty)$.

(2) $\sup \nu\left(A_{i}\right)=\nu\left(\sup A_{i}\right)$ for every sequence, $A_{1} \leq A_{2} \leq A_{3} \leq \cdots$, of elements of $K(\mathscr{H})$ with $\sup A_{i} \in K(\mathscr{H})$.

We adhere, in this definition, to the usual monotone continuous rules for adding and multiplying elements of $[0, \infty]$;

$$
\begin{gathered}
0 \cdot \infty=\infty \cdot 0=0, \\
a \cdot \infty=\infty \cdot a=\infty \quad \text { for all } a>0, \\
a+\infty=\infty+a=\infty \quad \text { for all } a \geq 0 .
\end{gathered}
$$


These rules imply $\nu(0)=0$ for any normal form $\nu$.

There is a useful connection between normal forms and the so-called "sesquilinear forms" in $\mathscr{H}$. Let $\mathscr{D}$ be a nonempty linear subset of $\mathscr{H}$. Following the usual convention [4], a symmetric (sesquilinear) form $f$ on $\mathscr{D}$ is a function $f: \mathscr{D} \times \mathscr{D} \rightarrow \mathbf{C}$ with $f(\psi, \varphi)=\overline{f(\varphi, \psi)}$, linear in the first argument and antilinear in the second argument. The symmetric form $f$ is nonnegative if $f(\varphi, \varphi) \geq 0$ for every $\varphi \in \mathscr{D}$. The nonnegative symmetric form $f$ is called closed if it behaves like $(\Phi \psi, \Phi \varphi)$, where $\Phi$ is a closed linear operator with domain, $\mathscr{D}$, i.e. if $\mathscr{D}$ is a Hilbert space in the inner product, $[\cdot, \cdot]$ defined by the expression

$$
[\psi, \varphi]:=f(\psi, \varphi)+(\psi, \varphi) \quad(\psi, \varphi \in \mathscr{D}) .
$$

Let $\nu$ be a normal form on $K(\mathscr{H})$. Defining, for $\varphi$ and $\psi$ elements of $\mathscr{H}$, the rank one linear operator

$$
E_{\varphi, \psi} \eta:=(\eta, \psi) \varphi \quad(\eta \in \mathscr{H}),
$$

on $\mathscr{H}$, consider the subset, $\mathscr{D}_{\nu}$, of those $\varphi \in \mathscr{H}$ with $\nu\left(E_{\varphi, \varphi}\right)<\infty$. $\mathscr{D}_{\nu}$ is a nonempty linear subset of $\mathscr{H}$ and $f_{\nu}(\varphi, \varphi):=\nu\left(E_{\varphi, \varphi}\right)$ defines, by means of the polarisation formula, a nonnegative symmetric form $f_{\nu}$ on $\mathscr{D}_{\nu}$. The observation that makes it possible to transfer all considerations to the cone of normal forms is the following lemma:

LEMMA 1.1. If $\nu: K(\mathscr{H}) \rightarrow[0, \infty]$ is a normal form on $K(\mathscr{H})$, then the symmetric form $f_{\nu}$ defined on $D_{\nu}$ is closed.

PROOF. The completeness of $\mathscr{D}_{\nu}$ in the norm of the inner product, $[\psi, \varphi]:=$ $f_{\mu}(\psi, \varphi)+(\psi, \varphi) ;\left(\psi, \varphi \in \mathscr{D}_{\nu}\right)$ is proved as follows: For $\psi \in \mathscr{H}$, let $|[\psi]|$ denote the $[\cdot, \cdot]$-norm of $\psi$. If $\psi_{1}, \psi_{2}, \psi_{3}, \ldots$ is a $|[\cdot]|$-Cauchy sequence in $\mathscr{D}_{\nu}$, we can choose a subsequence, also denoted by $\psi_{1}, \psi_{2}, \psi_{3}, \ldots$, such that

$$
0<\|\left[\psi_{i}-\psi_{j} \|^{2} \leq 1 / 2^{i} \quad(j>i)\right.
$$

for all $j$. The sequence $\left\{\psi_{i}\right\}$ is also a $\|\cdot\|$-Cauchy sequence and has a $\|\cdot\|$-limit, $\psi$, in $\mathscr{H}$. Let $a_{1}, a_{2}, \ldots$ be positive numbers with

$$
\sum_{k=1}^{\infty} a_{k}^{-2}<\infty, \quad \sum_{k=1}^{\infty} 2^{-k} a_{k}^{2}<\infty .
$$

For $\varphi \in \mathscr{H}, \varepsilon>0$, one has, for $i$ sufficiently large, $\left|\left(\psi_{i}, \varphi\right)\right|^{2} \geq|(\psi, \varphi)|^{2}-\varepsilon$. Setting $\psi_{0}:=0, \eta_{k}:=\psi_{k}-\psi_{k-1}$, so that $\psi_{n}=\eta_{1}+\eta_{2}+\cdots+\eta_{n}$ for all $n$, we have,

$$
\begin{aligned}
\left|\left(\psi_{i}, \varphi\right)\right|^{2} & =\left|\sum_{k=1}^{i}\left(\eta_{k}, \varphi\right)\right|^{2}=\left|\sum_{k=1}^{i} a^{2}\left(\eta_{k}, \varphi\right) a^{-2}\right|^{2} \\
& \leq \sum_{k=1}^{i} a_{k}^{-2} \sum_{k=1}^{i}\left|\left(\eta_{k}, \varphi\right)\right|^{2} a_{k}^{2}
\end{aligned}
$$

which gives

$$
|(\psi, \varphi)|^{2} \leq \sum_{k=1}^{\infty} a_{k}^{-2} \sum_{k=1}^{\infty}\left|\left(\eta_{k}, \varphi\right)\right|^{2} a_{k}^{2}
$$


This can be rewritten

$$
\left(E_{\psi, \psi} \varphi, \varphi\right) \leq \sum_{k=1}^{\infty} a_{k}^{-2} \sum_{k=1}^{\infty}\left(E_{\eta_{k}, \eta_{k}} \varphi, \varphi\right) a_{k}^{2}
$$

We consider the operator,

$$
C_{i}:=\sum_{k=1}^{i} a_{k}^{2} E_{\eta_{k}, \eta_{k}}
$$

We have

$$
\begin{aligned}
\left(C_{i} \varphi, \varphi\right) & =\sum_{k=1}^{i} a_{k}^{2}\left|\left(\eta_{k}, \varphi\right)\right|^{2} \leq \sum_{k=1}^{i} a_{k}^{2}\left\|\eta_{k}\right\|^{2}\|\varphi\|^{2} \\
& \leq\left(\sum_{k=1}^{\infty} a_{k}^{2}\left|\left[\eta_{k}\right]\right|^{2}\right)\|\varphi\|^{2}
\end{aligned}
$$

which shows that

$$
C_{\infty}:=\sum_{k=1}^{\infty} a_{k}^{2} E_{\eta_{k}, \eta_{k}}
$$

is an element of $K(\mathscr{H})$ with

$$
E_{\psi, \psi} \leq \sum_{k=1}^{\infty} a_{k}^{-2} C_{\infty}
$$

By monotone continuity of $\nu$,

$$
\begin{aligned}
\nu\left(E_{\psi, \psi}\right) & \leq \sum_{k=1}^{\infty} a_{k}^{-2} \nu\left(C_{\infty}\right) \\
& =\left(\sum_{k=1}^{\infty} a_{k}^{-2}\right) \sum_{k=1}^{\infty} a_{k}^{2} \nu\left(E_{\eta_{k}, \eta_{k}}\right) \\
& \leq\left(\sum_{k=1}^{\infty} a_{k}^{-2}\right) \sum_{k=1}^{\infty} a_{k}^{2} \mid\left[\eta_{k} \|^{2}\right. \\
& \leq\left(\sum_{k=1}^{\infty} a_{k}^{-2}\right) \sum_{k=1}^{\infty} a_{k}^{2} 2^{-k}<\infty
\end{aligned}
$$

which shows that $\psi \in \mathscr{D}_{\nu}$.

Repeating the above argument, we have

$$
E_{\psi-\psi_{i-1}, \psi-\psi_{i-1}} \leq \sum_{k=1}^{\infty} a_{k}^{-2} \sum_{k=i}^{\infty} a_{k}^{2} E_{\eta_{k}, \eta_{k}}
$$

because

$$
\sum_{k=i}^{n} \eta_{k} \stackrel{n}{\rightarrow} \psi-\psi_{i-1}
$$

Thus

$$
f_{\nu}\left(\psi-\psi_{i}, \psi-\psi_{i}\right) \leq \sum_{k=i}^{\infty} a_{k}^{-2} \sum_{k=i}^{\infty} a^{2}\left|\left[\eta_{k}\right]\right|^{2} \stackrel{i}{\rightarrow} 0
$$

as was to be shown. 
Let $\mathscr{D}$ be a nonempty linear subset of $\mathscr{H}, \bar{D}$ the closure of $\mathscr{D}$ in the norm of $\mathscr{H}$. If $f$ is a closed nonnegative symmetric form with domain of definition $\mathscr{D}$, then the second representation theorem [4, p. 331] asserts that there is a nonnegative selfadjoint operator $\Phi: \mathscr{D} \rightarrow \overline{\mathscr{D}}$ with $f(\varphi, \psi)=(\Phi \varphi, \Phi \psi)$ for all $\varphi$ and $\psi$ in $\mathscr{D}$. Using the uniqueness of polar decomposition, one sees that every nonnegative selfadjoint operator in the Hilbert space $\overline{\mathscr{D}}$ with domain $\mathscr{D}$ having this relation to $f$ is equal to $\Phi$.

Let $\mathscr{D}$ be a nonempty linear subset of $\mathscr{H}, f$ a closed nonnegative symmetric form with domain $\mathscr{D}$. Considering the above operator $\Phi$, we can write, using the spectral representation,

$$
(\Phi \varphi, \Phi \varphi)=\int_{[0, \infty)} \lambda^{2}(Z(d \lambda) \varphi, \varphi) \quad(\varphi \in \mathscr{D}),
$$

where $Z([0, \infty))$ is the projection onto $\overline{\mathscr{D}}$. This can be written

$$
(\Phi \varphi, \Phi \varphi)=\int_{[0, \infty)} \lambda^{2} \operatorname{tr}\left[E_{\varphi, \varphi} Z(d \lambda) E_{\varphi, \varphi]} \quad(\varphi \in \mathscr{D}) .\right.
$$

If $B \in K(\mathscr{H})$ then define the measure,

$$
m_{B}(E)=\operatorname{tr}\left[B^{1 / 2} Z(E) B^{1 / 2}\right]=\operatorname{tr}[Z(E) B Z(E)]
$$

on the Borel subsets $E \subset[0, \infty)$. Then, using the convention, $Z(\{\infty\})=I-$ $Z([0, \infty))$, we can extend the measure to the Borel sets of $[0, \infty]$, and define the normal form,

$$
\nu(B)=\int_{[0, \infty]} \lambda^{2} m_{B}(d \lambda) \quad(B \in K(\mathscr{H})),
$$

on $K(\mathscr{H})$ which is equal to $f(\varphi, \varphi)=(\Phi \varphi, \Phi \varphi)$ for $B=E_{\varphi, \varphi}(\varphi \in \mathscr{D})$ and for which $\mathscr{D}_{\nu}=\mathscr{D}$. Thus we have found a surjective and injective correspondence between the normal forms on $K(\mathscr{H})$ and the set of ordered pairs $(f, \mathscr{D})$, where $\mathscr{D}$ is a nonempty linear subset of $\mathscr{H}$, and $f$ is a closed nonnegative symmetric form with domain $\mathscr{D}$.

From the foregoing one sees that every normal form $\nu$ can be written in the form

$$
\nu(B)=\int_{[0, \infty]} \lambda \operatorname{tr}\left[B^{1 / 2} M(d \lambda) B^{1 / 2}\right] \quad(B \in K(\mathscr{H})),
$$

where $M$ is a spectral measure on $[0, \infty]$. Any spectral measure, $M$, defines a normal form $\nu$ in this way. Thus any $\nu$ can be approximated from below by finite sums in the sense,

$$
\nu(B)=\lim _{n} \sum_{i=1}^{n} \nu_{i}(B) \quad(B \in K(\mathscr{H})),
$$

where $\nu_{1}, \nu_{2}, \nu_{3}, \ldots$ are normal forms with $\mathscr{D}_{\nu_{i}}=\mathscr{H}$ for each $i$. The operator $\rho_{\nu}:=\int \lambda M(d \lambda)$ defined in $\overline{\mathscr{D}}_{\nu}$ is uniquely determined by $\nu$, being the square of $\Phi=\int \lambda Z(d \lambda)$, the operator representing $f_{\nu}$. 
2. General remarks on the cone of normal forms. The set $\mathscr{F}$ of normal forms is a cone over the extended nonnegative reals; if $\nu$ is a normal form and $\lambda \in[0, \infty]$, then $\lambda \nu(B)(B \in K(\mathscr{H}))$ defines a new normal form. There are two immediately obvious ways of introducing a semiorder in $\mathscr{F}$. The semiordering

$$
\mu \geq \nu \Leftrightarrow \mu(B) \geq \nu(B) \quad(B \in K(\mathscr{H}))
$$

will be called the weak semiordering. The semiordering induced by the additive structure

$$
\mu \geq \nu \Leftrightarrow \exists \omega \in \mathscr{F} \quad \text { with } \mu=\nu+\omega
$$

will be called the "additive" semiordering. In the cones usually under discussion, relevant alternatives to the additive semiordering are equivalent to it. This is not so for the weak semiordering in $\mathscr{F}$. To show this, we make use of a remark which is essentially due to T. Kato [4, p. 329].

REMARK 2.1. Any unbounded closed nonnegative symmetric form $f$ with domain $\mathscr{D}$ can be properly extended to a closed nonnegative symmetric form.

The boundedness of a symmetric form is that usually defined; $f$ on $\mathscr{D}$ is bounded iff there exists a $\lambda \in R$ with $f(\varphi, \varphi) \leq \lambda\|\varphi\|^{2}$ for all $\varphi \in \mathscr{D}$. This is equivalent to $\mathscr{D}$ being a closed linear subset of $\mathscr{H}$. Kato does not supply a proof in [4], nor does a proof seem to be readily available. We sketch one here.

PROOF OF THE REMARK. It suffices to consider the case where $\mathscr{D}$ is dense in $\mathscr{H}$ and where $f(\varphi, \varphi)>0$ for all $\varphi \in \mathscr{D}$. Suppose $f(\varphi, \psi)=(\Phi \varphi, \Phi \psi)(\varphi, \psi \in \mathscr{D})$, where $\Phi$ is a nonnegative selfadjoint operator in $\mathscr{H}$ with domain $\mathscr{D}$. The range of $\Phi$ is dense in $\mathscr{H}$. If $f$ is unbounded, then there is a $\eta \in \mathscr{H}$ not in $\mathscr{D}$, and the set, $\Delta$, of all $\varphi \in \mathscr{D}$ with $(\eta, \Phi \varphi)=0$ is dense in $\mathscr{H}$. Let $A=\Phi^{2}$. $A$ is also nonnegative and selfadjoint in $\mathscr{H}$. Consider the operator, $S=(I-A)(I+A)^{-1}$. The operator $A=(I-S)(I+S)^{-1}$ has domain equal to the range of $I+S$ so that $\Delta$, being contained in the domain of $\Phi=(I-S)^{1 / 2}(I+S)^{-1 / 2}$, is also in the domain of $(I+S)^{-1 / 2}$. Let $D=(I+S)^{-1 / 2} \Delta$ and let $S_{0}$ be the restriction of $S$ to $D$. Then $\left(I+S_{0}\right) D=(I+S) D=(I+S)^{1 / 2} \Delta$ and $\left(I+S_{0}\right) D$ is dense in $\mathscr{H}$, because $S$ has no eigenvalue equal to -1 . Thus $A_{0}=\left(I-S_{0}\right)\left(I+S_{0}\right)^{-1}$ is a densely defined nonnegative symmetric operator, and we can apply the results in connection with Theorem I, $\S 109$ in $[1]$. Since $(I+S)^{1 / 2} D=\Delta$ is dense in $\mathscr{H}$, the orthogonal projection, $Q_{1}$, onto the orthogonal complement of $\Delta$ is zero, and $(I+S)^{1 / 2} Q_{1}(I+S)^{1 / 2}=0$. Therefore, in the notation of $[1], A=A_{\mu}$, the nonnegative selfadjoint extension of $A_{0}$ in the sense of Friedrichs. But $(I-S)^{1 / 2} D=$ $\Phi \Delta$ is nondense in $\mathscr{H}$, being orthogonal to $\eta$, whereas $(I-S)^{1 / 2} \mathscr{H}$ is dense in $\mathscr{H}$, because it contains the range of $\Phi$. Thus $(I-S)^{1 / 2} Q_{2}(I-S)^{1 / 2} \neq 0$, where $Q_{2}$ is the orthogonal projection onto $(I-S)^{1 / 2} D$. Thus there is a nonnegative selfadjoint extension, $A_{M}$ of $A_{0}$, not equal to the extension, $A_{\mu}$, of Friedrichs. Using $A_{M}$, the remark now follows by an application of Krein's theorem and the lemma of Friedrichs (Theorem 2 and the immediately preceding lemma in $\S 109$ of [1]).

We can now show that if $\nu \in \mathscr{F}$ with $f_{\nu}$ unbounded, then there is a $\bar{\nu} \in \mathscr{F}$ such that $\bar{\nu}$ is weakly but not additively bounded by $\nu$. We can assume that $D_{\nu}$ is dense in $\mathscr{H}$. Let $f_{\bar{\nu}}$ be a proper extension of $f_{\nu}$, where $\bar{\nu} \in \mathscr{F}$. Then $\nu \neq \bar{\nu}$ with $\bar{\nu}(B) \leq \nu(B)(B \in K(\mathscr{H}))$. The normal form $\nu$ is not additively greater than $\bar{\nu}$. Otherwise there would be $\omega \in \mathscr{F}$ with $\nu=\bar{\nu}+\omega$. If $\Phi$ were the selfadjoint operator defined on $\mathscr{D}_{\omega}$ with $(\Phi \varphi, \Phi \varphi)=f_{\omega}(\varphi, \varphi)$ for all $\varphi \in \mathscr{D}_{\omega}$, then $\Phi$ would 
have to be zero on the dense subset $\mathscr{D}_{\nu}$ of $\mathscr{H}$, which would imply $\Phi=0$, therefore $\omega=0$ and $\nu=\bar{\nu}$, a contradiction.

REMARK 2.2. If $\nu$ and $\bar{\nu}$ are normal forms with $\mathscr{D}_{\bar{\nu}}=\mathscr{H}$ and $\bar{\nu} \leq \nu$ weakly then $\bar{\nu} \leq \nu$ additively.

ProOF. The nonnegative symmetric form, $f=f_{\nu}-f_{\bar{\nu}}$, defined on $\mathscr{D}_{\nu}$ is closed and defines a form $\omega \in \mathscr{F}$ with $\nu=\bar{\nu}+\omega$.

If $\nu_{1} \leq \nu_{2} \leq \nu_{3} \cdots$ is a weakly increasing sequence of normal forms, then their weak supremum exists in $\mathscr{F}$. This is not always so for the corresponding statement in terms of the additive semiordering: If $\nu \geq \bar{\nu}$ weakly but not additively, then consider a sequence of normal forms, $\bar{\nu}_{1}, \bar{\nu}_{2}, \bar{\nu}_{3}, \ldots$ with $\bar{\nu}=\sum \bar{\nu}_{i}$ and $\mathscr{D}_{\bar{\nu}_{i}}=\mathscr{H}$ for each $i$. The form $\bar{\nu}$ is obviously an additive bound for each finite sum. Therefore a form which could serve as an additive supremum for the set of finite sums would have to be identical to $\bar{\nu}$. But $\bar{\nu}$ can not be an additive supremum, because $\nu$, which bounds each finite sum additively, does not bound $\bar{\nu}$ additively.

3. Remarks on positive linear maps on $\mathscr{F}$. Let $\mathscr{F}_{b}$ be the subset of elements, $\nu \in \mathscr{F}$, with $\mathscr{D}_{\nu}=\mathscr{H}$. Considering $\mathscr{F}$ as a cone over the reals, $\mathscr{F}_{b}$ is a subcone of $\mathscr{F}$. The map, $G: K(\mathscr{H}) \rightarrow \mathscr{F}_{b}$ defined by

$$
[G(A)](B)=\operatorname{tr}\left[A^{1 / 2} B A^{1 / 2}\right]=\operatorname{tr}\left[B^{1 / 2} A B^{1 / 2}\right]=[G(B)](A) \quad(A, B \in K(\mathscr{H}))
$$

is positive linear on $K(\mathscr{H})$ and monotone continuous there, i.e. if $A_{1} \leq A_{2} \leq$ $A_{3} \cdots$ is a sequence of elements of $K(\mathscr{H})$ with $\sup A_{i} \in K(\mathscr{H}), \operatorname{then} G\left(\sup A_{i}\right)=$ sup $G\left(A_{i}\right)$ in the sense of the weak semiordering in $\mathscr{F}$, and $G^{-1}: \mathscr{F}_{b} \rightarrow K(\mathscr{H})$ is also positive linear on $\mathscr{F}_{b}$ and monotone continuous there. Thus every $\nu \in \mathscr{F}$ is, by means of $\mu \rightarrow \nu\left(G^{-1}(\mu)\right)\left(\mu \in \mathscr{F}_{b}\right)$, an extended real-valued function on $\mathscr{F}_{b}$, positive linear and monotone continuous in the sense already defined for $G$. One has

$$
\begin{aligned}
\nu\left(G^{-1}(\mu)\right) & =\left[G\left(G^{-1}(\nu)\right)\right]\left(G^{-1}(\mu)\right)=\left[G\left(G^{-1}(\mu)\right)\right]\left(G^{-1}(\nu)\right) \\
& =\mu\left(G^{-1}(\nu)\right)
\end{aligned}
$$

for $\mu, \nu \in \mathscr{F}_{b}$. The function, $\nu \cdot G^{-1}$ on $\mathscr{F}_{b}$, can easily be extended to all of $\mathscr{F}$. Write

$$
\nu\left(G^{-1}(\mu)\right)=\sum_{i} \nu_{i}\left(G^{-1}(\mu)\right) \quad\left(\mu \in \mathscr{F}_{b}\right)
$$

where $\nu_{i} \in \mathscr{F}_{b}$ for all $i$. Then define

$$
\bar{\nu}(\mu)=\sum_{i} \mu\left(G^{-1}\left(\nu_{i}\right)\right) \quad(\mu \in \mathscr{F}) .
$$

If $\mu \in \mathscr{F}_{b}$, then $\mu\left(G^{-1}\left(\nu_{i}\right)\right)=\nu_{i}\left(G^{-1}(\mu)\right)$ for each $i$, which shows that the restriction of $\bar{\nu}$ to $\mathscr{F}_{b}$ is equal to $\nu \cdot G^{-1}$. We collect the properties of $\bar{\nu}$ in a definition.

DEFINITION. A normal form $\bar{\nu}$ on $\mathscr{F}$ is an extended real-valued function from $\mathscr{F}$ into $[0, \infty]$ with the following properties with respect to the weak semiordering in $\mathscr{F}$ :

(i) $\alpha, \beta \in[0, \infty] ; \mu, \omega \in \mathscr{F} \Rightarrow \bar{\nu}(\alpha \mu+\beta \omega)=\alpha \bar{\nu}(\mu)+\beta \bar{\nu}(\omega)$.

(ii) $\mu \geq \omega \Rightarrow \bar{\nu}(\mu) \geq \bar{\nu}(\omega)(\mu, \omega \in \mathscr{F})$.

(iii) If $\mu_{1} \leq \mu_{2} \leq \mu_{3} \leq \cdots$ is a sequence of elements of $\mathscr{F}$, then $\bar{\nu}\left(\sup \mu_{i}\right)=$ $\sup \bar{\nu}\left(\mu_{i}\right)$.

The set of normal forms on $\mathscr{F}$ is a cone over $[0, \infty]$ in the obvious sense, and 
will be denoted by $\overline{\mathscr{F}}$. It is clear that an extension of $\nu \cdot G^{-1}: \mathscr{F}_{b} \rightarrow[0, \infty]$, with given $\nu \in \mathscr{F}$, to a normal form on $\mathscr{F}$ is uniquely determined. The correspondence $\nu \rightarrow \mu \cdot G^{-1} \rightarrow \bar{\nu}$ from $\mathscr{F}$ to $\bar{F}$ is injective and also surjective because $\bar{\nu}$ is the extension of its restriction to $\mathscr{F}_{b}$, and this restriction is positive linear and monotone continuous there, thus of the form $\nu \cdot G^{-1}$. The semiordering in $\overline{\mathscr{F}}$ defined by

$$
\bar{\nu} \geq \bar{\omega} \Leftrightarrow \bar{\nu}(\mu) \geq \bar{\omega}(\mu) \quad(\mu \in \mathscr{F}),
$$

is related to the weak semiordering in $\mathscr{F}$ by

$$
\nu \geq \omega \Leftrightarrow \bar{\nu} \geq \bar{\omega}
$$

which becomes apparent if we write the correspondence $\nu \leftrightarrow \bar{\nu}$ between the elements of $\mathscr{F}$ and those of $\overline{\mathscr{F}}$ in another way: Let $\mu \in \mathscr{F}$ and $\mu=\sum \mu_{i}$ with $\mu_{i} \in \mathscr{F}_{b}$ for every $i$. Then

$$
\begin{aligned}
\bar{\nu}(\mu)= & \sum_{i} \sum_{j} \mu_{j}\left(G^{-1}\left(\nu_{i}\right)\right) \\
& =\sum_{j} \sum_{i} \nu_{i}\left(G^{-1}\left(\mu_{j}\right)\right)=\sum_{j} \nu\left(G^{-1}\left(\mu_{j}\right)\right) .
\end{aligned}
$$

It is now also apparent that the correspondence $\nu \leftrightarrow \bar{\nu}$ has, in both directions, the properties (i) and (iii), (interpreted in the appropriate sense) as well, and that $\bar{\nu}(\mu)=\bar{\mu}(\nu)$ for all $\mu$ and $\nu$ in $\mathscr{F}$. There is an alternative characterisation for the elements of $\overline{\mathscr{F}}$.

DEFINITION. Let $\bar{g}: \mathscr{F} \rightarrow[0, \infty]$ be a positive linear function on $\mathscr{F}$. We call $\bar{g}$ $\sigma$-additive if, for every sequence, $\nu_{1}, \nu_{2}, \nu_{3}, \ldots$ of elements of $\mathscr{F}$,

$$
\bar{g}\left(\sum \nu_{i}\right)=\sum \bar{g}\left(\nu_{i}\right)
$$

Every element of $\overline{\mathscr{F}}$ is obviously $\sigma$-additive, but the converse is also true; every positive linear $\sigma$-additive function $\bar{g}: \mathscr{F} \rightarrow[0, \infty]$ is an element of $\overline{\mathscr{F}}$, because the restriction, $g$, of $\bar{g}$ to $\mathscr{F}_{b}$ is positive linear there and monotone continuous, the weak and additive semiordering being equivalent in $\mathscr{F}_{b}$. The extension of $g$ to an element of $\overline{\mathscr{F}}$ is, like $g, \sigma$-additive and therefore equal to $\bar{g}$, there being only one $\sigma$-additive extension of $g$ to $\mathscr{F}$. Thus $g \in \overline{\mathscr{F}}$.

We call a map $T: \mathscr{F} \rightarrow \mathscr{F}$ positive linear if $T(\alpha \mu+\beta \nu)=\alpha T \mu+\beta T \nu$ for all $\alpha$ and $\beta$ in $[0, \infty]$ and all $\mu$ and $\nu$ in $\mathscr{F}$. We call $T$ monotone if $\mu \geq \nu$ in the weak sense implies $T \mu \geq T \nu$, also in the weak sense. If $T$ is positive linear and monotone, then we call $T$ monotone continuous if, for every weakly monotone sequence, $\nu_{1} \leq \nu_{2} \leq \nu_{3} \leq \cdots$, of elements of $\mathscr{F}, \sup T \nu_{i}=T\left(\sup \nu_{i}\right)$, also in the sense of the weak semiordering.

DEFINITION. A positive linear map $T: \mathscr{F} \rightarrow \mathscr{F}$ is $\sigma$-additive if, for every sequence, $\nu_{1}, \nu_{2}, \nu_{3}, \ldots$, of elements of $\mathscr{F}$

$$
T\left(\sum \nu_{i}\right)=\sum T \nu_{i}
$$

REMARK 3.1. A positive linear map $T: \mathscr{F} \rightarrow \mathscr{F}$ is monotone and monotone continuous if and only if it is $\sigma$-additive. 
Proof. If $T$ is $\sigma$-additive, then it is also monotone: Let $\mu \geq \nu$ in the weak sense, and let $\nu=\sum \nu_{i}$ where, for each $i, \mathscr{D}_{\nu_{i}}=\mathscr{H}$. Since $\mu$ is additively greater than each finite sum, $\sum \nu_{i}$, then

$$
T \mu \geq T\left(\sum_{i=1}^{n} \nu_{i}\right)=\sum_{i=1}^{n} T \nu_{i}
$$

and

$$
T \mu \geq \lim _{n} \sum_{i=1}^{n} T \nu_{i}=\sum_{i=1}^{\infty} T \nu_{i}=T\left(\sum \nu_{i}\right)=T \nu,
$$

by $\sigma$-additivity.

Let $\bar{\nu}$ be an arbitrary element of $\overline{\mathscr{F}}$. Then $\bar{\nu} \cdot T$ is $\sigma$-additive on $\mathscr{F}$, therefore also an element of $\overline{\mathscr{F}}$. If $\nu_{1} \leq \nu_{2} \leq \nu_{3} \leq \cdots$ is a weakly monotone sequence of elements of $\mathscr{F}$, then

$$
\begin{aligned}
\bar{\nu}\left(T\left(\sup \nu_{i}\right)\right) & =(\bar{\nu} \cdot T)\left(\sup \nu_{i}\right)=\sup (\bar{\nu} \cdot T)\left(\nu_{i}\right) \\
& =\sup \bar{\nu}\left(T \nu_{i}\right)=\bar{\nu}\left(\sup T \nu_{i}\right),
\end{aligned}
$$

because $T \nu_{1}, T \nu_{2}, T \nu_{3}, \ldots$ is weakly monotone. Since $\bar{\nu}$ is arbitrary, $T\left(\sup \nu_{i}\right)=$ $\sup T \nu_{i}$ and $T$ is monotone continuous.

4. The operator inequality in the general setting. Let $\phi:[0, \infty] \rightarrow[0, \infty]$ be an extended valued measurable function and $\nu \in \mathscr{F}$. Then we define $\phi(\nu)$ to be the normal form such that $\rho_{\phi(\nu)}=\phi\left(\rho_{\nu}\right)$, where $\rho_{\nu}(\nu \in \mathscr{F})$ was defined at the end of $\S 2$. If $\phi:[0, \infty] \rightarrow[-\infty, \infty]$ is an arbitrary extended valued measurable function, then we define $\phi(\nu)(\nu \in \mathscr{F})$ only if one of the normal forms, $\phi_{+}(\nu)$ or $\phi_{-}(\nu)$ is finite valued on $K(\mathscr{H})$. Then we say that $\phi(\nu)$ exists and is equal to $\phi_{+}(\nu)-\phi_{-}(\nu)$. The theorem of Lindblad now assumes the following more general form. We denote the normal form on $K(\mathscr{H})$ defined by the trace by $\tau$.

THEOREM 4.1. Let $T: \mathscr{F} \rightarrow \mathscr{F}$ be a positive linear and $\sigma$-additive transformation with

$$
\begin{aligned}
{[T \nu](I) } & =\nu(I) ; \quad(\nu \in \mathscr{F}), \\
T \tau & =\tau .
\end{aligned}
$$

If $\phi:[0, \infty) \rightarrow(-\infty, \infty)$ is a convex function and $\nu \in \mathscr{F}$ with $\nu(I)<\infty$, then $\left[\phi_{+}(T \nu)\right](I)$ is finite if $\left[\phi_{+}(\nu)\right](I)$ is. If $\phi(\nu)$ and $\phi(T \nu)$ both exist, then $[\phi(T \nu)](I)$ $\leq[\phi(\nu)](I)$.

PROOF. Since $\nu(I)$ and $[T \nu](I)$ are finite there are orthogonal bases, $\psi_{1}, \psi_{2}$, $\psi_{3}, \ldots$ and $\eta_{1}, \eta_{2}, \eta_{3}, \ldots$ for $\mathscr{H}$ and normal forms, $\alpha_{1}, \alpha_{2}, \ldots, \beta_{1}, \beta_{2}, \ldots$, such that

$$
\nu=\sum x_{i} \alpha_{i}, \quad T \nu=\sum \lambda_{j} \beta_{j}
$$

where $x_{i}$ and $\lambda_{j}$ are nonnegative real numbers for each $i$ and $j$, where

$$
\alpha_{i}\left(E \psi_{j}, \psi_{j}\right)=\beta_{i}\left(E \eta_{j}, \eta_{j}\right)=\left\{\begin{array}{ll}
1 & \text { for } j=i \\
0 & \text { for } j \neq i
\end{array} \quad(\text { all } i, j)\right.
$$

and where, for any $f:[0, \infty) \rightarrow[0, \infty)$,

$$
f(\nu)=\sum f\left(x_{i}\right) \alpha_{i}, \quad f(T \nu)=\sum f\left(\lambda_{j}\right) \beta_{j} .
$$


Then $\sum x_{i} T \alpha_{i}=\sum \lambda_{j} \beta_{j}$, and

$$
\lambda_{j}=\sum_{i} x_{i}\left[T \alpha_{i}\right]\left(E_{\eta_{j}, \eta_{j}}\right) \quad(\text { all } j) .
$$

Since $\sum \alpha_{i}=\tau$, then $\sum T \alpha_{i}=T(\tau)=\tau$, and

$$
\sum_{i}\left[T \alpha_{i}\right]\left(E_{\eta_{j}, \eta_{j}}\right)=\tau\left(E_{\eta_{j}, \eta_{j}}\right)=1
$$

Since $\phi_{+}$is convex, Jensen's inequality gives

$$
\begin{aligned}
{\left[\phi_{+}(T \nu)\right](I) } & =\sum \phi_{+}\left(\lambda_{j}\right) \leq \sum \phi_{+}\left(x_{i}\right)\left[T \alpha_{i}\right](I) \\
& =\left[T\left(\sum \phi_{+}\left(x_{i}\right) \alpha_{i}\right)\right](I) \\
& =\left[\sum \phi_{+}\left(x_{i}\right) \alpha_{i}\right](I)=\left[\phi_{+}(\nu)\right](I) .
\end{aligned}
$$

For the proof of the second statement of the theorem, the existence of $\phi(T \nu)$ implies that either $\sum \phi_{+}\left(\lambda_{j}\right)$ or $\sum \phi_{-}\left(\lambda_{j}\right)$ is finite. Thus the sum $\sum \phi\left(\lambda_{j}\right)$ is defined and equal to $[\phi(T \nu)](I)$. The existence of $\phi(\nu)$ implies that either $\sum \phi_{+}\left(x_{i}\right) \alpha_{i}$ or $\sum \phi_{-}\left(x_{i}\right) \alpha_{i}$ is finite valued on $K(\mathscr{H})$, therefore that either

$$
\sum_{i, j} \phi_{+}\left(x_{i}\right)\left[T \alpha_{i}\right]\left(E_{\eta_{j}, \eta_{j}}\right) \quad \text { or } \quad \sum_{i, j} \phi_{-}\left(x_{i}\right)\left[T \alpha_{i}\right]\left(E_{\eta_{j}, \eta_{j}}\right)
$$

is finite. Thus the sum

$$
\sum_{j} \sum_{i} \phi\left(x_{i}\right)\left[T \alpha_{i}\right]\left(E_{\eta_{j}, \eta_{j}}\right)
$$

is well enough defined for repeating the above proof with $\phi_{+}$replaced by $\phi$.

\section{REFERENCES}

1. N. I. Akhiezer and I. M. Glazman, Theory of linear operators in Hilbert space, Pitman, Boston, Mass., London, and Melbourne, 1980.

2. C. C. Brown, Entropy increase as a consequence of measure invariance, Proc. Amer. Math. Soc. 93 (1985), 448-450.

3. J. Dixmier, Les algèbres d'opérateurs dans l'espace hilbertien (Algèbres de von Neumann), deuzième éd., Gauthier-Villars, 1969.

4. T. Kato, Perturbation theory for linear operators, Springer, Berlin and New York, 1966.

5. G. Lindblad, Expectations and entropy inequalities for quantum systems, Comm. Math. Phys. 39 (1974), 111-120.

INStitut FÜr MAThematik, III Freie UNIVERSität, ARNimAllee 2-6, 1000 Berlin 33, Federal Republic of Germany 